\section{Gossypiboma successfully removed by endoscopy after endoscopic ultrasound-guided transmural drainage}
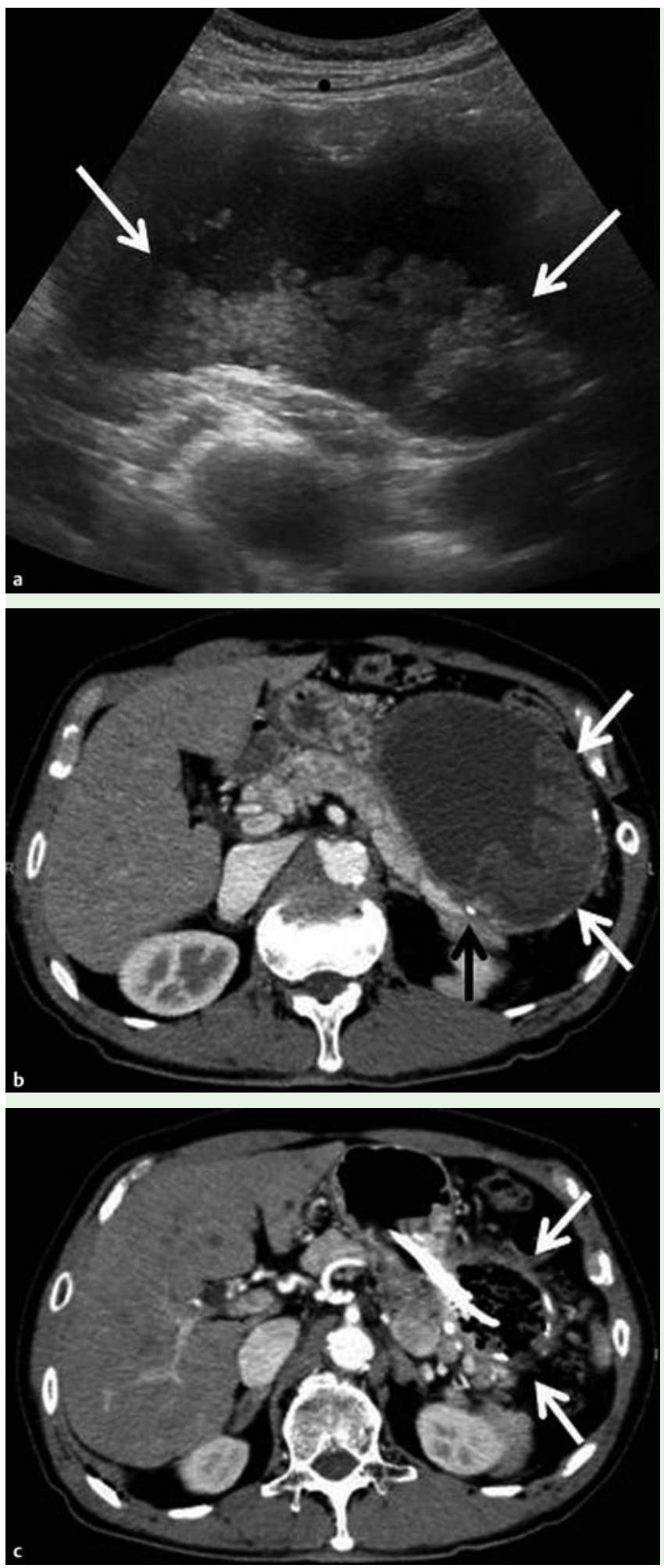

Fig. 1 a Ultrasound image in a 77-year-old man with abdominal delineated cystic mass containing internal high-echoic irregular mass-like debris (white arrows). $\mathbf{b}$ Computed tomography (CT) scan showing a well-delineated, 12-cm cystic mass with a whorled appearance (white arrow) with calcification of the wall (black arrow). c CT scan (after the EUS-guided drainage) shows the decrease in the cyst size to $5 \mathrm{~cm}$ and change to an internal spongiform appearance with scattered gas bubbles (white arrow). pain showing a well-
Endoscopy is an emerging diagnostic as well as treatment modality for gossypiboma [1-5]. Here we report successful endoscopic removal of a gossypiboma after endoscopic ultrasound (EUS)-guided transmural drainage.

A 77-year-old man presented with abdominal pain. He had undergone an operation 30 years ago for alcohol-induced esophageal varices. Ultrasound (US) showed a well-delineated cystic mass containing internal high-echoic irregular mass-like debris ( Fig.1a). Abdominal computed tomography (CT) revealed a well-defined, 12-cm cystic mass, with internal whorled appearance and wall calcification, in contact with the pancreas and stomach ( $\bullet$ Fig.1b), which was diagnosed as a pancreatic pseudocyst. We chose to puncture the cystic mass transgastrically with a 19-G needle (Echo-tip Ultra; Cook Medical, Bloomington, Indiana, USA) under EUS guidance, following which we inserted two 7-Fr double pigtail-type plastic stents (Olympus Medical Systems, Tokyo, Japan). About 1 month later, the diameter of the cyst decreased from $12 \mathrm{~cm}$ to $5 \mathrm{~cm}$ and it showed an altered spongiform internal appearance with scattered gas bubbles on CT ( Fig. 1 c). We considered the possibility of a gossypiboma, and an upper gastrointestinal endoscopy revealed surgical gauze protruding from the side of the plastic stents ( Fig.2a). We carried out balloon dilation (Hurricane RX, $10 \mathrm{~mm}$ wide dilation, $4 \mathrm{~cm}$ length; Boston Scientific, Natick, Massachusetts, USA) of the fistula ( Fig. 2b) and using biopsy forceps, the gauze was fully extracted slowly without any complications ( Fig.2c and - Video 1).

To our knowledge, this is the first reported case of a gossypiboma managed with endoscopy after EUS-guided transmural drainage. The gauze did not migrate into the lumen in our patient, but a pseudocyst was formed in response to long-term inflammation. After EUS-guided cyst drainage, the gauze protruded from the side of the inserted plastic stents, and we were able to remove it by transgastric endoscopy. We believe that this method may become a common treatment for gossypiboma in patients in whom the material has not migrated.

\section{Video 1}

Endoscopic removal of surgical gauze. 

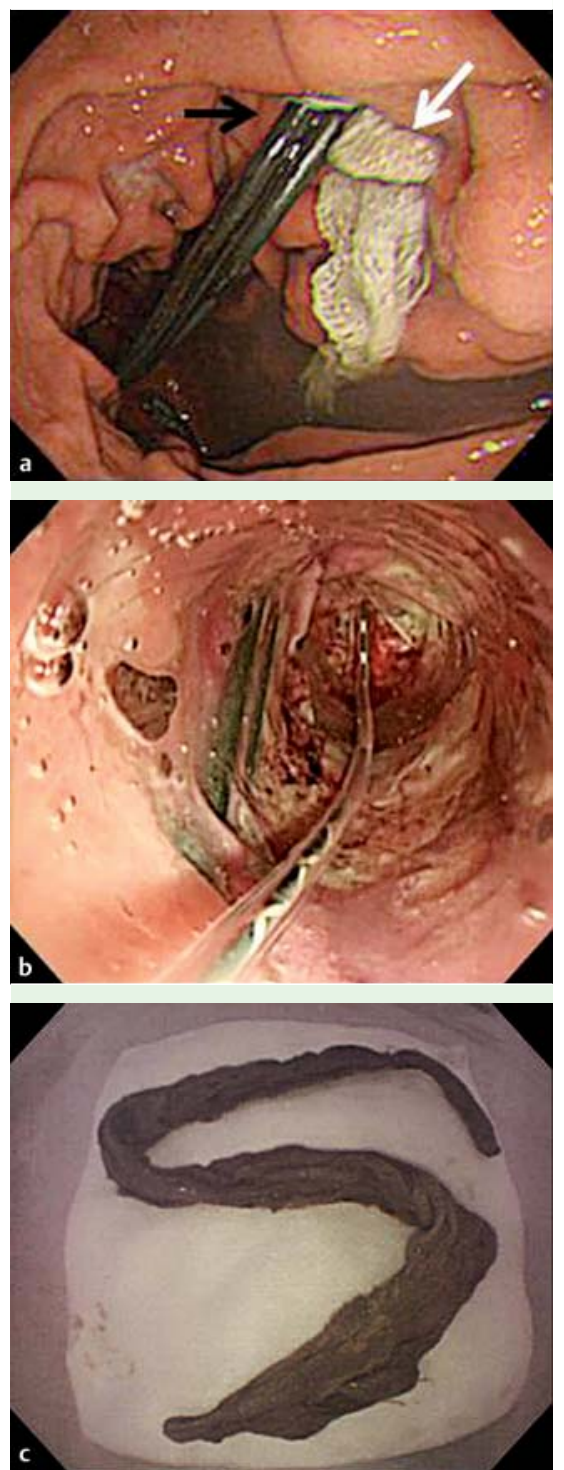

Fig. 2 a A piece of surgical gauze (white arrow) protruding from the side of the stents (black arrow) at the stomach. b Endoscopic balloon dilation of the fistula between the stomach and the cyst. $\mathbf{c}$ The surgical gauze after endoscopic removal.
Endoscopy_UCTN_Code_CCL_1AF_2AG_3AD

\section{Competing interests: None}

\section{K. Matsumoto, A. Katanuma,}

H. Maguchi, K. Takahashi, M. Osanai, K. Yane, T. Kin, R. Takaki, T. Matsumori, K. Gon, A. Tomonari

Center for Gastroenterology,

Teine-Keijinkai Hospital, Hokkaido, Japan

\section{References}

1 Erbay G, Koc Z, Caliskan $K$ et al. Imaging and clinical findings of a gossypiboma migrated into the stomach. Turk J Gastroenterol 2012; 23: 54-57

2 Saroj K, Harsh P, Thakur D et al. Gossypiboma diagnosed by upper-G1 endoscopy. Gastrointest Endosc 2007; 65: 347-349

3 Cipolletta L, Bianco MA, Rotondano G et al. Endoscopic retrieval of a surgical gauze from the common bile duct. Ital J Gastroenterol Hepatol 1997; 29: 58-61
4 Hinrichs C, Methratta S, Ybasco AC et al. Gossypiboma treated by colonoscopy. Pediatr Radiol 2003; 33: 261 - 262

5 Pevrin-Biroulet L, Oliver A, Biqard MA et al. Gossypiboma successfully removed by upper-GI endoscopy. Gastrointest Endosc 2007; 6: $1251-1252$

Bibliography

DOI http://dx.doi.org/

10.1055/s-0033-1344326

Endoscopy 2013; 45: E212-E213

(c) Georg Thieme Verlag KG

Stuttgart · New York

ISSN 0013-726X

\section{Corresponding author}

\section{K. Matsumoto}

Center for Gastroenterology

Teine-Keijinkai Hospital

1-jo 12-chome

Maeda

Teine-ku

Sapporo 006-8555

Japan

Fax: +81-11-6852967

matsumotokazuyuki0227@yahoo.co.jp 